\title{
On the implementation of the Biological Threat Reduction Program in the Republic of Uzbekistan
}

\author{
Laziz Tuychiev*1 and Marifjon Madaminov² \\ ${ }^{1}$ Sanitary-Epidemiological Department of Ministry of Health, Tashkent, Uzbekistan; ${ }^{2}$ Center for Prophilaxis and Quarantine of Most \\ Hazardous Infections of Uzbekistan, Tashkent, Uzbekistan
}

\section{Objective}

To review the implementation of the Biological Threat Reduction Program (BTRP) of the U.S. Defense Threat Reduction Agency in the Republic of Uzbekistan since 2004

\section{Introduction}

The Biological Threat Reduction Program (BTRP) has been being implemented in the Republic of Uzbekistan since 2004 within the framework of the Agreement between the Government of the Republic of Uzbekistan and the Government of the United States of America Concerning Cooperation in the Area of the Promotion of Defense Relations and the Prevention of Proliferation of Weapons of Mass Destruction of 06.05.2001. Threat agent detection and response activities that target a list of especially dangerous pathogens are being carried out under the BTRP within the health care system of Uzbekistan. This presentation reviews some of the achievements of the program to date.

\section{Results}

BTRP, in partnership with the Government of Uzbekistan, has funded the establishment of five Regional Diagnostic Laboratories (RDL) and ten Epidemiological Support Units (ESU), operated by the Ministry of Health of Uzbekistan, which are intended to improve the diagnosis of quarantine and especially dangerous infections, and to ensure timely preventive and anti-epidemic measures.

RDLs provide a high level of biosafety and biosecurity to conduct rapid laboratory diagnostics (PCR, ELISA) of especially dangerous infections. RDLs are equipped with up-to-date diagnostic laboratory equipment that conforms to internationals standards, as well as with all necessary consumables.

Personnel of RDLs have been appropriately trained in epidemiology, clinical and diagnostic techniques for especially dangerous infections, including such state-of-the-art techniques as rapid PCR and
ELISA diagnostics, as well as in work and equipment operation safety regulations.

Epidemiological Support Units (ESU) have been established on the basis of the Especially Dangerous Infections Divisions of Oblast, city and Rayon Centers for State Sanitary and Epidemiological Surveillance (SES) of the Ministry of Health. The BTRP ESU efforts include renovation activities, supply and installation of appropriate equipment for rapid laboratory diagnostics, and vehicles.

ESUs are meant to ensure emergency notification in cases of suspected occurrence of quarantine and especially dangerous infections, for timely implementation of anti-epidemic and preventive measures.

Three Cooperative Biological Research projects on quarantine and especially dangerous infections have been implemented within BTRP with the financial support of the US Civilian Research and Development Foundation (CRDF).

To ensure the sustainability of training and availability of a pool of skilled personnel, a training laboratory is to be established at the Tashkent Institute for Post-Graduate Medical Education (TIPME) to train personnel of RDLs and ESUs. The training laboratory will fully replicate the setup of a BSL-2 Regional Diagnostic Laboratory, but will maintain no operations with live pathogens.

\section{Conclusions}

The implementation of BTRP within the health care system of the Republic of Uzbekistan contributes to the stable and sustainable wellbeing in the population of the country.

\section{Keywords}

Biological Threat Reduction Program; Especially dangerous pathogens; Resource-poor setting

*Laziz Tuychiev

E-mail: laziz.tyuchiev@minzdrav.uz 